VoL. 47 (1993) [247-257]

\title{
ON THE GENERALISED RUSCHEWEYH CLASS OF ANALYTIC FUNCTIONS OF COMPLEX ORDER
}

\author{
O.P. AHUJA
}

The main object of this paper is to identify various classes of analytic functions which are starlike, convex, pre-starlike, Ruscheweyh class of order $\alpha, \beta$-spirallike, $\beta$-convex-spiral-like, starlike of complex order, complex of complex order, and others as special cases of a family of analytic functions of complex order in the unit disk. This makes a uniform treatment possible. Finally, we derive sharp estimates for all coefficients of the functions from the family.

\section{INTRODUCTION AND DEFINITIONS}

Let $\mathbb{A}$ denote the family of functions $f$ which are analytic in the unit disk $\triangle=$ $\{z:|z|<1\}$ and normalised such that $f(0)=0=f^{\prime}(0)-1$. Denote by $S$ the subfamily of $\mathbb{A}$ consisting of the functions that are univalent in $\triangle$.

An analytic function $f$ is said to be subordinate to an analytic function $g$ (written $f \ll g)$ if $f(z)=g(\omega(z)), z \in \Delta$, for some analytic function $\omega$ with $\omega(0)=0$ and $|\omega(z)|<1$ in $\triangle$. The Hadamard product of two power series

$$
f(z)=\sum_{k=0}^{\infty} a_{k} z^{k}, \quad g(z)=\sum_{k=0}^{\infty} b_{k} z^{k}
$$

is defined as the power series

$$
(f * g)(z)=\sum_{k=0}^{\infty} a_{k} b_{k} z^{k}, \quad z \in \triangle .
$$

Denote $D^{\lambda}: \mathbb{A} \rightarrow \mathbb{A}$ the operator defined by

$$
D^{\lambda} f=\frac{z}{(1-z)^{\lambda+1}} * f, \quad \lambda \geqslant-1, \quad z \in \triangle .
$$

Notice that $d^{-1} f=z, D^{0} f=f, D^{1} f=z f^{\prime}, D^{2} f=z\left(f^{\prime}+z f^{\prime \prime} / 2\right)$. Let $H_{\lambda}^{b}[A, B]$ denote the class of functions $f$ in $\mathbb{A}$ satisfying the condition

$$
1+\frac{(\lambda+1)}{b}\left(\frac{D^{\lambda+1} f}{D^{\lambda} f}-1\right) \ll \frac{1+A z}{1+B z},-1 \leqslant B<A \leqslant 1, z \in \Delta,
$$

Received 10 March 1992

Copyright Clearance Centre, Inc. Serial-fee code: 0004-9729/93 \$A2.00+0.00. 
where $\lambda>-1$ and $b \neq 0$ is an arbitrary fixed complex number. We call $H_{\lambda}^{b}[A, B]$ the generalised Ruscheweyh class of analytic functions of complex order $b$. This is due to the fact that the class

$$
K_{\lambda}:=\left\{f \in \mathbb{A}: \operatorname{Re}\left(\frac{D^{\lambda+1} f}{D^{\lambda} f}\right)>1 / 2, z \in \Delta\right\} \equiv H_{\lambda}^{(1+\lambda) / 2}[1,-1]
$$

was first introduced and studied by Ruscheweyh [22].

A function $f \in \mathbb{A}$ is said to be in $S[A, B]$ if $\left(z f^{\prime} / f\right) \ll(1+A z) /(1+B z)$, and in $K[A, B]$ if $\left(1+z f^{\prime \prime} / f^{\prime}\right) \ll(1+A z) /(1+B z)$ for some arbitrary fixed real numbers $A$ and $B(-1 \leqslant B<A \leqslant 1)$ and for all $z$ in $\triangle$. Such classes were investigated in [12, 13, 14, 26, 27], and others. In view of (1.1) and (1.2), it follows that

$$
\begin{gathered}
f \in S[A, B] \Leftrightarrow f \in H_{0}^{1}[A, B], \\
f \in K[A, B] \Leftrightarrow f \in H_{1}^{1}[A, B] .
\end{gathered}
$$

For $\alpha, \alpha \leqslant 1$, let

$$
\begin{aligned}
& S T(\alpha):=\left\{f \in \mathbb{A}: \operatorname{Re}\left(z f^{\prime} / f\right)>\alpha, z \in \Delta\right\} \\
& C V(\alpha):=\left\{f \in \mathbb{A}: \operatorname{Re}\left(1+z f^{\prime \prime} / f^{\prime}\right)>\alpha, z \in \triangle\right\}
\end{aligned}
$$

The functions in $S T(\alpha)$ and $C V(\alpha)$ are called, respectively, starlike of order $\alpha$ and convex of order $\alpha$ in $\triangle$. It is well known that $C V(\alpha) \subset S T(\alpha) \subset S$ whenever $0 \leqslant \alpha \leqslant 1$. We observe that for $\alpha<1$,

$$
S T(\alpha) \equiv H_{0}^{1-\alpha}[1,-1], \quad C V(\alpha) \equiv H_{1}^{1-\alpha}[1,-1]
$$

In fact, by setting $b=1$ and for different choices of $A, B(-1 \leqslant B<A \leqslant 1)$, the class $H_{\lambda}^{b}[A, B]$ will give rise to various subclasses of $S T(0)$; for instance, the class

$$
H_{0}^{1}[1,-1+1 / \alpha] \equiv\left\{f \in \mathbb{A}:\left|z f^{\prime} / f-\alpha\right|<\alpha, z \in \Delta\right\}, \quad \alpha>1 / 2 .
$$

was introduced in [30]. For other such classes which may be obtained from $H_{\lambda}^{b}[A, B]$, one may refer to $[19,26,27]$.

Other larger sets are formed by $\beta$-spiral-like functions of order $\alpha$, denoted by $S P(\beta ; \alpha)$, and $\beta$-Robertson (or $\beta$-convex spiral-like) functions of order $\alpha$, denoted by $R B(\beta ; \alpha)$. Here $\alpha$ and $\beta$ are arbitrary fixed real numbers satisfying the conditions $-\pi / 2<\beta<\pi / 2$ and $0 \leqslant \alpha<1$. These classes were introduced, respectively, in [16] and $[9]$. We recall that

$$
\begin{aligned}
& S P(\beta ; \alpha):=\left\{f \in \mathbb{A}: \operatorname{Re}\left(e^{i \beta} z f^{\prime} / f\right)>\alpha \cos \beta, \quad z \in \Delta\right\} \\
& R B(\beta ; \alpha):=\left\{f \in \mathbb{A}: \operatorname{Re} e^{i \beta}\left(1+z f^{\prime \prime} / f^{\prime}\right)>\alpha \cos \beta, \quad z \in \Delta\right\}
\end{aligned}
$$


Equivalent conditions are

$$
\begin{aligned}
& f \in S P(\beta ; \alpha) \Leftrightarrow f \in H_{0}^{e^{-i \beta} \cos \beta}[1-2 \alpha,-1] \\
& f \in R B(\beta ; \alpha) \Leftrightarrow f \in H_{1}^{e^{-i \beta} \cos \beta}[1-2 \alpha,-1] .
\end{aligned}
$$

Incidently, by fixing $b=e^{-i \beta} \cos \beta,|\beta|<\pi / 2$ and $\lambda=0$, we may obtain several known subclasses of $S P(\beta ; 0)$ from the family $H_{\lambda}^{b}[A, B]$; for instance, for $-1 \leqslant B<$ $A \leqslant 1, \alpha>1 / 2, c+d \geqslant 1, d \leqslant c \leqslant d+1$, we have

$$
\begin{aligned}
& H_{0}^{e^{-i \beta} \cos \beta}[A, B] \equiv\left\{f \in \mathbb{A}: e^{i \beta} z f^{\prime} / f \ll \frac{1+A z}{1+B z} \cos \beta+i \sin \beta, z \in \Delta\right\}, \\
& H_{0}^{e^{-i \beta} \cos \beta}[1,-1+1 / \alpha] \equiv\left\{f \in \mathbb{A}:\left|e^{i \beta} z f^{\prime} / f-(\alpha \cos \beta+i \sin \beta)\right|\right. \\
& <\alpha \cos \beta, z \in \triangle\}
\end{aligned}
$$

$$
H_{0}^{e^{-i \beta} \cos \beta}\left[\frac{d^{2}-c^{2}+c}{d}, \frac{1-c}{d}\right] \equiv\left\{f \in \mathbb{A}:\left|e^{i \beta} z f^{\prime} / f-(c \cos \beta+i \sin \beta)\right|\right.
$$

$$
<d \cos \beta, z \in \Delta\} \text {. }
$$

Denote the classes defined in (1.10) to (1.12), respectively, by $\mathbb{S P}(\beta ; A, B), \mathbb{S} \mathbb{P}(\beta ; \alpha)$ and $\mathbb{S P}[\beta ; c, d]$. These classes were introduced and studied in $[10],[15]$ and $[29]$, respectively. Analogous subclasses of $R B(\beta ; 0)$, denoted by $\mathbb{R} B(\beta ; A, B), \mathbb{R} B(\beta ; \alpha)$, and $\mathbb{R} \mathbb{B}[\beta ; c, d]$, may be obtained by fixing $b=e^{-i \beta} \cos \beta, \lambda=1$ and choosing appropriate values of $A$ and $B$. In fact, several such classes studied in $[2,4,11,17,18,21]$ may be related to the family $H_{\lambda}^{b}[A, B]$.

Let $\mathbb{R}_{\alpha}$ be the class of pre-starlike functions of order $\alpha$; that is, $f \in \mathbb{R}_{\alpha}$ if and only if

$$
\begin{cases}f * \frac{z}{(1-z)^{2-2 \alpha}} \in S T(\alpha), & \alpha<1 \\ \operatorname{Re}(f(z) / z)>1 / 2 & \alpha=1, z \in \Delta .\end{cases}
$$

Note that

$$
s_{\alpha}(z)=z /(1-z)^{2-2 \alpha}
$$

is a well-known extremal function in $S T(\alpha)$. Ruscheweyh [23] observed that

$$
f \in \mathbb{R}_{\alpha} \Leftrightarrow \operatorname{Re}\left(D^{2-2 \alpha} f / D^{1-2 \alpha} f\right)>1 / 2, z \in \Delta, \alpha \leqslant 1
$$

Equivalently,

$$
f \in \mathbb{R}_{\alpha} \Leftrightarrow f \in H_{1-2 \alpha}^{1-\alpha}[1,-1], \alpha<1
$$


It is known [28] that $\mathbb{R}_{\alpha} \subset S$ if and only if $\alpha \leqslant 1 / 2$.

Given $\alpha<1$ and $\beta<1$, a function $f$ in $A$ is said to be pre-starlike of order $\alpha$ and type $\beta, f \in \mathbb{R}(\alpha, \beta)$, if $f * s_{\alpha} \in S T(\beta)$. Note that $\mathbb{R}(\alpha, \alpha) \equiv \mathbb{R}_{\alpha}, \alpha<1$. The class $\mathbb{R}(\alpha, \beta)$ was investigated in [25]. Using (1.1) and the identity

$$
z\left(D^{\lambda} f\right)^{\prime}=(\lambda+1) D^{\lambda+1} f-\lambda D^{\lambda} f, \quad \lambda>-1 .
$$

we may write

$$
\mathbb{R}(\alpha, \beta)=\left\{f \in \mathbb{A}: \operatorname{Re}\left(z\left(D^{1-2 \alpha} f\right)^{\prime} / D^{1-2 \alpha} f\right)>\beta, z \in \Delta\right\},
$$

and thus it follows that

$$
f \in \mathbb{R}(\alpha, \beta) \Leftrightarrow f \in H_{1-2 \alpha}^{1-\beta}[1,-1], \alpha<1, \beta<1 .
$$

Let $\mathbb{R}_{\lambda}(\alpha)$ denote the class of functions $f$ in $\mathbb{A}$ satisfying the condition

$$
\operatorname{Re} \frac{z\left(D^{\lambda} f(z)\right)^{\prime}}{D^{\lambda} f(z)}>\alpha
$$

for some $\lambda>-1, \alpha<1$, and for all $z \in \Delta$. We may call $\mathbb{R}_{\lambda}(\alpha)$ the Ruscheweyh class of order $\alpha$. In view of (1.3) and the identity (1.15), we have $\mathbb{R}_{\lambda}((1-\lambda) / 2) \equiv K_{\lambda}$ for $\lambda>-1$. For $\lambda=n \in\{0,1,2, \ldots\}$ and $0 \leqslant \alpha<1$, the classes $\mathbb{R}_{n}(0)$ and $\mathbb{R}_{n}(\alpha)$ were, respectively, introduced in [31] and [1]. Also, note that $D^{n} f=\left(z\left(z^{n-1} f\right)^{(n)}\right) / n$ !, $n \in\{0,1,2, \ldots\}$. Finally, we observe that

$$
f \in \mathbb{R}_{\lambda}(\alpha) \Leftrightarrow f \in H_{\lambda}^{1-\alpha}[1,-1], \alpha<1, \lambda>-1 .
$$

and

$$
\mathbb{R}_{\lambda}(\alpha) \equiv H_{\lambda}^{1}[1-\alpha,-1] \equiv H_{\lambda}^{1-\alpha}[1,-1], 0 \leqslant \alpha<1 .
$$

Some such classes involving the Ruscheweyh derivatives and related to $H_{\lambda}^{b}[A, B]$ were studied in $[3,5,6,7,24]$.

Aouf and Nasr [8] introduced the class $S_{b}$ of starlike functions of complex order $b\left(b \neq 0\right.$ and complex); that is, $f \in S_{b}$ if and only if

$$
\operatorname{Re}\left(1+\frac{1}{b}\left(\frac{z f^{\prime}(z)}{f(z)}-1\right)\right)>0, z \in \Delta .
$$

An analogous class $C_{b}$ of complex order $b$ is defined by replacing $z f^{\prime} / f$ in (1.19) by $\left(1+z f^{\prime \prime} / f^{\prime}\right)$; see [8]. Equivalently, we have

$$
\begin{aligned}
& f \in S_{b} \Leftrightarrow f \in H_{0}^{b}[1,-1] \\
& f \in C_{b} \Leftrightarrow f \in H_{1}^{b}[1,-1] .
\end{aligned}
$$


In view of the relations witnessed in (1.3) to (1.12), (1.14), (1.16) to (1.18), (1.20) and (1.21), we conclude that the notion of the generalised Ruscheweyh class, $H_{\lambda}^{b}[A, B]$, of analytic functions of complex order $b$, unifies several subclasses of $\mathbb{A}$. Thus a study of $H_{\lambda}^{b}[A, B]$ may lead to information about $K_{\lambda}, S[A, B], K[A, B]$, $S T(\alpha), C V(\alpha), S P(\beta ; \alpha), R B(\beta ; \alpha), \mathbb{S P}(\beta ; A, B), \mathbb{S P}(\beta ; \alpha), \mathbb{S P}[\beta ; c, d], \mathbb{R} \mathbb{R}(\beta ; A, B)$, $\mathbb{R} \mathbb{R}(\beta ; \alpha), \quad \mathbb{R} \mathbb{B}[\beta ; c, d], \mathbb{R}_{\alpha}, \mathbb{R}(\alpha, \beta), \mathbb{R}_{\lambda}(\alpha), S_{b}, C_{b}$, and those obtained by choosing $\lambda \geqslant 2$ and suitable values of the parameters $b, A$ and $B$. Finally, we obtain sharp coefficient estimates of the functions in $H_{\lambda}^{b}[A, B]$.

\section{Coefficient estimates}

Lemma 1. [20] Let

$$
g(z)=\sum_{p=q}^{\infty} d_{p} z^{p}, \quad G(z)=\sum_{p=q}^{\infty} D_{p} z^{p}, \quad q \geqslant 0 .
$$

If $g(z)=\omega(z) G(z)$ where $\omega(0)=0$ and $|\omega(z)|<1$ in $\Delta$, then $d_{q}=0$ and

$$
\sum_{p=q+1}^{k}\left|d_{p}\right|^{2} \leqslant \sum_{p=q}^{k-1}\left|D_{p}\right|^{2}, \quad(k=q+1, q+2, \ldots) .
$$

Lemma 2. For a fixed integer $m, m \geqslant 3$, let

$$
M_{j}=\frac{|(A-B) b-(j-2) B|^{2}}{(\lambda+j-1)^{2}}, \quad(j=2,3, \ldots, m),
$$

and $\quad C(\lambda, p)=\frac{(\lambda+1)_{p-1}}{(p-1) !}=\frac{(\lambda+1)(\lambda+2) \ldots(\lambda+p-1)}{(p-1) !} \quad(p=2,3, \ldots)$.

Then

$$
\begin{gathered}
\left(\frac{1}{(m-1) C(\lambda, m)}\right)^{2}\left((A-B)^{2}|b|^{2}+\sum_{p=2}^{m-1}\left\{|(A-B) b-(p-1) B|^{2}\right.\right. \\
\left.\left.-(p-1)^{2}\right\}(C(\lambda, p))^{2} \prod_{j=2}^{p} M_{j}\right)=\prod_{j=2}^{m} M_{j} .
\end{gathered}
$$

ProOF: We shall prove (2.1) by mathematical induction on $m$. A brief calculation shows that (2.1) holds for $m=3$. Assume that (2.1) is valid for $m=4,5, \ldots, t-1$; 
then for $m=t$, the left side of (2.1) gives

$$
\begin{gathered}
\left(\frac{1}{(t-1) C(\lambda, t)}\right)^{2}\left((A-B)^{2}|b|^{2}+\sum_{p=2}^{t-2}\left\{|(A-B) b-(p-1) B|^{2}-(p-1)^{2}\right\}(C(\lambda, p))^{2}\right. \\
\left.\prod_{j=2}^{p} M_{j}+\left\{|(A-B) b-(t-2) B|^{2}-(t-2)^{2}\right\}(C(\lambda, t-1))^{2} \prod_{j=2}^{t-1} M_{j}\right) \\
=\left(\frac{1}{(t-1) C(\lambda, t)}\right)^{2}\left(((t-2) C(\lambda, t-1))^{2} \prod_{j=2}^{t-1} M_{j}+\left\{(\lambda+t-1)^{2} M_{t}\right.\right. \\
\left.\left.-(t-2)^{2}\right\}(C(\lambda, t-1))^{2} \prod_{j=2}^{t-1} M_{j}\right)=\prod_{j=2}^{t} M_{j} .
\end{gathered}
$$

This concludes the proof of (2.1)

Theorem. Let $f \in H_{\lambda}^{b}[A, B]$, and $f(z)=z+\sum_{k=2}^{\infty} a_{k} z^{k}, z \in \Delta$. Then

$$
\left|a_{2}\right| \leqslant \frac{(A-B)|b|}{\lambda+1}
$$

and if $|(A-B) b-B| \leqslant 1$ and $k \geqslant 3$, then

$$
\left|a_{k}\right| \leqslant \frac{(A-B)|b|(k-2) !}{(\lambda+1)_{k-1}}
$$

Furthermore, if $|(A-B) b-(k-2) B|>(k-2), k \geqslant 3$, let

$$
M=\left[\frac{|(A-B) b-(k-2) B|}{k-2}\right]
$$

be the greatest integer less than or equal to the expression within the square bracket. Then

$$
\left|a_{k}\right| \leqslant\left(1 /(\lambda+1)_{k-1}\right) \prod_{j=2}^{k}|(A-B) b-(j-2) B|
$$

for $k=3,4, \ldots, M+2$; and

$$
\left|a_{k}\right| \leqslant \frac{(k-2) !}{(M+1) !(\lambda+1)_{k-1}} \prod_{j=2}^{M+3}|(A-B) b-(j-2) B|
$$


for $k>M+2$. The bounds in (2.2) to (2.4) are sharp for all admissible $b, \lambda, A, B$, and for each $k$.

Proof: From (1.2), we have

$$
1+\frac{\lambda+1}{b}\left(\frac{D^{\lambda+1} f}{D^{\lambda} f}-1\right)=\frac{1+A \omega}{1+B \omega}, \quad z \in \Delta
$$

for some analytic function $\omega$ with $\omega(0)=0$ and $|\omega(z)|<1$ in $\Delta$. On simplification, (2.6) yields

$$
(\lambda+1)(1+B \omega) D^{\lambda+1} f=\{\lambda+1+\{(A-B) b+(\lambda+1) B\} \omega\} D^{\lambda} f
$$

that is,

$$
(\lambda+1)\left(D^{\lambda+1} f-D^{\lambda} f\right)=\left(\{(A-B) b+(\lambda+1) B\} D^{\lambda} f-B(\lambda+1) D^{\lambda+1} f\right) \omega(z) .
$$

Since

$$
D^{\lambda} f=z+\sum_{p=2}^{\infty} C(\lambda, p) a_{p} z^{p}
$$

and $C(\lambda+1, p)=((\lambda+p) /(\lambda+1)) C(\lambda, p)$, it follows that (2.7) is equivalent to

$$
\sum_{p=2}^{\infty}(p-1) C(\lambda, p) a_{p} z^{p}=\left(\sum_{p=1}^{\infty}\{(A-B) b-(p-1) B\} C(\lambda, p) a_{p} z^{p}\right) \omega(z)
$$

where $a_{1}=1$. Using Lemma 1 , we have

$$
\sum_{p=2}^{k}(p-1)^{2}(C(\lambda, p))^{2}\left|a_{p}\right|^{2} \leqslant \sum_{p=1}^{k-1}|(A-B) b-(p-1) B|^{2}((C(\lambda, p)))^{2}\left|a_{p}\right|^{2}
$$

which simplifies to

$$
\begin{aligned}
\left|a_{k}\right|^{2} \leqslant & \left(\frac{1}{(k-1) C(\lambda, k)}\right)^{2}\left((A-B)^{2}|b|^{2}\right. \\
& \left.+\sum_{p=2}^{k-1}\left\{|(A-B) b-(p-1) B|^{2}-(p-1)^{2}\right\}(C(\lambda, p))^{2}\left|a_{p}\right|^{2}\right)
\end{aligned}
$$

for every $k=2,3, \ldots$ For $k=2$, we have

$$
\left|a_{2}\right|^{2} \leqslant\left(\frac{(A-B)|b|}{\lambda+1}\right)^{2}
$$


which proves (2.2). Suppose $|(A-B) b-B| \leqslant 1$ and $k \geqslant 3$. Then it follows that

$$
|(A-B) b-(k-2) B| \leqslant k-2, \quad k \geqslant 3 .
$$

Since all the terms under the summation in (2.8) are non-positive, we obtain

$$
\left|a_{k}\right| \leqslant \frac{(A-B)|b|}{(k-1) C(\lambda, k)}, \quad k \geqslant 3
$$

which establishes (2.3). However, if

$$
|(A-B) b-(k-2) B|>(k-2), \quad k \geqslant 3
$$

then all the terms under the summation in (2.8) are positive. We shall establish (2.4) for integer $k \geqslant 3$ and $k \leqslant M+2$, from (2.8), by mathematical induction. For $k=3$, (2.8) contributes

$$
\left|a_{3}\right|^{2} \leqslant\left(\frac{(A-B)|b||(A-B) b-B|}{(\lambda+1)(\lambda+2)}\right)^{2}
$$

which proves (2.4) for $k=3$. Suppose (2.4) holds for $k=4,5, \ldots, m-1$. Then for $k=m,(2.8)$ yields

$$
\begin{aligned}
\left|a_{m}\right|^{2} \leqslant & \left(\frac{1}{(m-1) C(\lambda, m)}\right)^{2}\left((A-B)^{2}|b|^{2}\right. \\
& \left.+\sum_{p=2}^{m-1}\left\{|(A-B) b-(p-1) B|^{2}-(p-1)^{2}\right\}(C(\lambda, p))^{2}\left|a_{p}\right|^{2}\right) \\
\leqslant & \left(\frac{1}{(m-1) C(\lambda, m)}\right)^{2}\left((A-B)^{2}|b|^{2}+\sum_{p=2}^{m-1}\left\{|(A-B) b-(p-1) B|^{2}\right.\right. \\
& \left.\left.-(p-1)^{2}\right\}(C(\lambda, p))^{2} \prod_{j=2}^{p} \frac{|(A-B) b-(j-2) B|^{2}}{(\lambda+j-1)^{2}}\right) \\
= & \prod_{j=2}^{m} \frac{|(A-B) b-(j-2) B|^{2}}{(\lambda+j-1)^{2}}
\end{aligned}
$$

by Lemma 2. It is now easy to show that (2.4) holds for $k \leqslant M+2$. Finally, suppose 
$k>M+2$. Then we may write $(2.8)$ as

$$
\begin{aligned}
\left|a_{k}\right|^{2} \leqslant & \left(\frac{1}{(k-1) C(\lambda, k)}\right)^{2}\left((A-B)^{2}|b|^{2}+\sum_{p=2}^{M+2}\left\{|(A-B) b-(p-1) B|^{2}\right.\right. \\
& \left.-(p-1)^{2}\right\}(C(\lambda, p))^{2}\left|a_{p}\right|^{2}+\sum_{p=M+s}^{k-1}\left\{|(A-B) b-(p-1) B|^{2}\right. \\
& \left.\left.-(p-1)^{2}\right\}(C(\lambda, p))^{2}\left|a_{p}\right|^{2}\right) \\
\leqslant & \left(\frac{1}{(k-1) C(\lambda, k)}\right)^{2}\left((A-B)^{2}|b|^{2}+\sum_{p=2}^{M+2}\left\{|(A-B) b-(p-1) B|^{2}\right.\right. \\
& \left.\left.-(p-1)^{2}\right\}(C(\lambda, p))^{2}\left|a_{p}\right|^{2}\right) \\
\leqslant & \left(\frac{1}{(k-1) C(\lambda, k)}\right)^{2}\left((A-B)^{2}|b|^{2}+\sum_{p=2}^{M+2}\left\{|(A-B) b-(p-1) B|^{2}\right.\right. \\
& \left.\left.\quad-(p-1)^{2}\right\}(C(\lambda, p))^{2} \prod_{j=2}^{p} \frac{|(A-B) b-(j=2) B|^{2}}{(\lambda+j-1)^{2}}\right) \\
= & \left(\frac{(M+2) C(\lambda, M+3)}{(k-1) C(\lambda, k)}\right)^{2} \prod_{j=2}^{M+3} \frac{|(A-B) b-(j-2) B|^{2}}{(\lambda+j-1)^{2}} \\
= & \left(\frac{(k-2) !}{(M+1) !(\lambda+1)(\lambda+2) \ldots(\lambda+k-1)}\right)^{2} \prod_{j=2}^{M+3}|(A-B) b-(j-2) B|^{2}
\end{aligned}
$$

by an application of Lemma 2 ; and thus (2.5) immediately follows from the above. The equality in (2.2) and (2.3) are attained for the functions $f_{k}$ given by

$$
f_{k} * \frac{z}{(1-z)^{\lambda+1}}= \begin{cases}z\left(1+B z^{k-1}\right)^{(A-B) b / B(k-1)}, & B \neq 0 \\ z \exp \left(A b z^{k-1} /(k-1)\right), & B=0\end{cases}
$$

where $|(A-B) b-B| \leqslant 1$. Finally, the inequality (2.4) is sharp for the function $f$ given by

$$
f * \frac{z}{(1-z)^{\lambda+1}}= \begin{cases}z(1+B z)^{(A-B) b / B}, & B \neq 0, \\ z \exp (A b z), & B=0,\end{cases}
$$

where $|(A-B) b-(k-2) B|>k-2, k \geqslant 3$.

REMARKs. On choosing appropriate values of the parameters $b, \lambda, A, B$ in the above Theorem, we may obtain the sharp estimates for all coefficients of the functions in several subclasses of $\mathbf{A}$. For instance, using the relations (1.3) to (1.6), (1.8) to (1.12), 
(1.14), (1.16), (1.18), (1.20) and (1.21), the Theorem will yield the corresponding coefficient estimates of the functions in the classes $K_{\lambda}, S[A, B], K[A, B], S T(\alpha), C V(\alpha)$, $S P(\beta ; \alpha), R B(\beta, \alpha), \mathbb{S P}(\beta ; A, B), \mathbb{S P}(\beta ; \alpha), \mathbb{S P}[\beta ; c, d], \mathbb{R}_{\alpha}, \mathbb{R}(\alpha, \beta), \mathbb{R}_{\lambda}(\alpha), S_{b}$ and $C_{b}$, respectively. In particular, the above Theorem generalises the results for the classes studied in $[8,10,12,16,18,26]$ and several others.

\section{REFERENCES}

[1] O.P. Ahuja, 'Integral operators of certain univalent functions', Internat. J. Math. Math. Sci. 8 (1985), 653-662.

[2] O.P. Ahuja, 'Certain generalizations of the Robertson functions', Yokohama Math. J. 31 (1983), 5-11.

[3] O.P. Ahuja and H. Silverman, 'Function classes related to Ruscheweyh derivatives', $J$. Austral. Math. Soc. Ser. A 47 (1989), 438-444.

[4] O.P. Ahuja and H. Silverman, 'A survey on spiral-like and related function classes', Math. Chronicle 20 (1991), 39-66.

[5] O.P. Ahuja and H. Silverman, 'Convolutions of prestarlike functions', Internat. J. Math. Math. Sci. 6 (1983), 59-68.

[6] H.S. Al-Amiri, 'On Ruscheweyh derivatives', Ann. Polon. Math. 38 (1980), 87-94.

[7] H.S. Al-Amiri, 'Certain generalizations of prestarlike functions', J. Austral. Math. Soc. Ser. A 28 (1979), 325-334.

[8] M.K. Aouf and M.A. Nasr, 'Starlike functions of complex order', J. Natur. Sci. Math. 25 (1985), 1-12.

[9] P.N. Chichra, 'Regular functions $f(z)$ for which $z f^{\prime}(z)$ is a-spiral-like', Proc. Amer. Math. Soc. 49 (1975), 151-160.

[10] Dasrath and S.L. Shukla, 'Coefficient estimates for a subclass of spiral-like functions', Indian J. Pure Appl. Math. 14 (1983), 431-439.

[11] R.M. Goel, 'A subclass of $\alpha$-spiral functions', Publ. Math. Debrecen 23 (1976), 79-84.

[12] R.M. Goel and B.S. Mehrok, 'On the coefficients of a subclass of starlike functions', Indian J. Pure Appl. Math. 12 (1981), 634-647.

[13] W. Janowski, 'Some extremal problems for certain families of analytic functions', Bull. de. L'Acad. Pol. des Sci. 21 (1973), 17-25.

[14] W. Janowski, 'Some extremal problems for certain families of analytic functions', Ann. Poln. Math. 28 (1973), 297-326.

[15] P.K. Kulshrestha, 'Bounded Robertson functions', Rend. Mat. 6 (1976), 137-150.

[16] R.J. Libera, 'Univalent $\alpha$-spiral functions', Canad. J. Math. 19 (1967), 449-456.

[17] B. Makowka, 'On some subclasses of univalent functions', Zeszyty Nauk. Politech Lódz. Math. 254 (1976), 71-76.

[18] M.L. Mogra and O.P. Ahuja, 'On spiral-like functions of order $\alpha$ and type $\beta$ ', Yokohama Math. J. 29 (1981), 145-156.

[19] K.S. Padmanabhan, 'On certain classes of starlike functions in the unit disk', $J$. Indian 
Math. Soc. 32 (1968), 89-103.

[20] M.S. Robertson, 'Quasi subordinate functions', in Mathematical Essays dedicated to A.J. MacIntyre Volume (Ohio University, 1967), pp. 311-340.

[21] M.S. Robertson, 'Univalent functions for which $z f^{\prime}(z)$ is spiral-like', Michigan Math. J. 16 (1969), 97-101.

[22] S. Ruscheweyh, 'New criteria for univalent functions', Proc. Amer. Math. Soc. 49 (1975), 109-115.

[23] S. Ruscheweyh, 'Linear operators between classes of prestarlike functions', Comment. Math. Helv. 52 (1977), 497-509.

[24] A. Schild and H. Silverman, 'Convolutions of univalent functions with negative coeffcients', Ann. Univ. Marie Curie-Sklodowska Sect. A 29 (1975), 99-107.

[25] T. Sheil-Small, H. Silverman and E.M. Silvia, 'Convolution multipliers and starlike functions', J. Analyse Math. 41 (1982), 181-192.

[26] H. Silverman, 'Subclasses of starlike functions', Rev. Roumaine Math. Pures Appl. 23 (1978), 1093-1099.

[27] H. Silverman and E.M. Silvia, 'Subclasses of starlike functions subordinate to convex functions', Canad. J. Math. 37 (1985), 48-61.

[28] H. Silverman and E.M. Silvia, 'Prestarlike functions with negative coefficients', Internat. J. Math. Math. Sci. 2 (1979), 427-439.

[29] E.M. Silvia, 'Subclasses of spiral-like functions', Tamkang J. Math. 14 (1983), 161-169.

[30] R. Singh, 'On a class of starlike functions II', Ganita 19 (1968), 103-110.

[31] R. Singh and S. Singh, 'Integrals of certain univalent functions', Proc. Amer. Math. Soc. 77 (1979), 336-343.

Department of Mathematics

The University of Papua New Guinea

Box 320, University Post Office

Papua New Guinea 